\title{
Assessment of allostasis and of the allostatic load as a control tool of the training
}

\author{
José Manuel GARCÍA-GARCÍA*, Guillermo LAICH, \& Bibiana CALVO-RICO \\ Faculty of Sport Science. Castilla-La Mancha University (Spain)
}

\section{Introduction}

The training aimed to participate successfully in competitions of high performance is a constant internal struggle of our body looking for a supercompensation functional system that bears it in order to improve our performance in each of the designed tasks. The organism perceives this task as an aggression (stress) that should bear, interpret and adapt positively to withstand the next charge. At different stages of annual training, the intensity and volume of the load is sometimes higher than what our body is able to bear, interpret and adapt, causing an imbalance in our homeostasis that can take us from technical malfunctions to an injury with no apparent reason and even getting a disease.

The "homeostasis" was a term introduced by the physiologist Walter Cannon in 1929. It is referred to a balance or harmony of systems and physiological parameters that allow a little variation such as body temperature, $\mathrm{pH}$, and arterial oxygen tension, glucose, protein, sodium, and calcium. On it, the physiology is regulated around strict values of a "set point" precisely determined. Therefore the stability of the internal environment is maintained by constancy in maintaining the set values or "set point" of their different systems and parameters. For these values to be remained settled it is needed to maintain their internal elements balance. These elements maintain homoeostasis and they are able to make the body react properly to different focal of aggression such as sports training, form the basis of the allostesis.

\section{Discussion and conclusion}

The allostasis concept focuses on the brain functions as the interpreter and reactive elements of the general and specific demands of the physical training, as well as the target organ of those demands.

The evolution of the central and peripheric nervous system, as well as of the neuroendocrine system, supplies an organizational principle to interpret the meaning of the adaptation mechanisms to stress and its relation to physical training. High performance athletes sometimes train indiscriminately, without load control, which can lead to an adaptative imbalance of the nervous system (Bear \& col, 1996)

Through allostasis the autonomous nervous system, the axle HPA, and the cardiovascular, immunological and metabolic systems protects the organism as a response to the physical training produced stress (McEwen, 2000). In a short time (acute allostatic load) these system is essential for the adaptation maintenance of allostasis and also to ease up of the function which means the same as to adequate the adaptative response to the training. In a long time (chronical allostatic load) an overload is created at a high physical and psychological cost which can accelerate and/or create several changes in the recovery processes.

Laich (2007) said that "allostasis" or allostatic regulation corresponds to a fairly new concept whose designation comes from Greek and the difference to homeostasis means "the ability to keep

*Email: ЏoseManuel.Garcia@uclm.es 
a stable internal environment upon change". Thus, allostasis concerns the ability of achieving stability through stress adaptation. This is due to the fact that adaptation allostatic systems are able to change, like biological buffers, so that the homeostasis systems which do not allow many changes can remain stable.

The allostatic response of a human being faced with a stressful factor is not always the same. In fact, there could be two very different forms: the "winners" are in the first place, the ones who train correctly and who have a positive and active response. In the second place come the "losers": sports people who do not train correctly, and neither control the loads and organization of their sessions bearing thus a negative and passive attitude towards the solutions to the allostatic loads.

In our popular slang, coaches often speak this way: "this judoka is a good competitor,". Although their technical skills are not very effective, and conversely, we ensure that a certain judoka, although in practice in the gym denote a high quality, "will not become champion because he/she is not a good competitor." It is more likely that this last instance has a problem with its stress management. Currently there are workouts designed for high performance athletes which aim is to focus on the athlete responses only on optimizing their resources.

But chronic allostatic state comes when programming inappropriately the loads and also when taking bad decisions related to the type and time of rest and especially in non-formed coaches, to inconsistency adaptive that produces certain workouts understudied and unscientific.

Finally, and trying to deep into the evils of our sports ,How many times have we seen our athletes ruined an optimal training program for a competition because of the suffer of variety in their weight the need to loose for a competition that has varied enormously the microcycle "finetuning"? Indeed, it has ceased to fine-tune his explosiveness and changed this objective by lipolytic aerobic training aspects, dehydrated and eating scarce nutrients that allow you to properly train... This process which is unfortunately common in our sports produces allostatic load a major limiting technical expressions and conditional expressions during competition, preventing optimize results the efforts made throughout the season.

Knowing how to deal correctly with the tools that allow us to control when a training load is becoming a chronical allostatic load, will benefit the sportsman training control, as well as the adequate development of the sporting progress.

\section{References}

Bear, M., Connors,W., \& Paradiso, M. (2006) Neuroscience, exploring the brain. Baltimore, MD: Lippincott Williams \& Wilkins.

Laich, G. (2005). Your fear is my power: the fine line between courage and cowardice. United Kingdom: SKM Publications.

Laich, G. (2007) Performance and psychiatric aspects of four world class athletes. Conference at the Congreso Internacional de la Asociación Americana de Psiquiatría (APA). Washington, D.C., EEUU.

McEwen, B. (2000) The neurobiology of stress: from serendipity to clinical relevance. Brain Research, 886(1-1), 172-189.

Key words: Combat sports; allostatic state; training control. 